\title{
Personal Display Wall
}

\section{Problem Statement}

Data created by modern supercomputing simulations offer a huge dynamic range of spatial scales. For example, simulations employing a technique called Adaptive Mesh Refinement (AMR), can offer a dynamic range of over 1 billion to 1 between the size of the largest displayable feature and the smallest. However, the highest resolution monitors currently available are incapable of capturing this dynamic range due to their limited resolution. Many fine details are lost due to the limited capabilities of the display medium.

The visualization community has adopted an approach that employs many displays that are arranged in a matrix in order to form "display walls" that offer the resolution necessary to properly display these multiscale visual structures (see references below). The display walls are typically very large and expensive - requiring special facilities to house them and considerable expertise to assemble and operate them. Likewise, there is a considerable amount of software development required to create effective applications on the commodity clusters that are typically employed to drive these display walls. It is very difficult to perform any useful development work when, due to cost and space considerations, the display wall is offered as a shared resource. There is a critical need for a smaller, simpler, less-expensive approach to creating high-resolution display walls that require very little expertise or labor to assemble and operate.

\section{Approach}

The LBNL Visualization Group has created a tiled display wall design that uses components that are readily available from a local hardware store and/or multiple online vendors, and requires minimal tools and skill to assemble (see Figure 1). The result is a low-cost, easy to assemble tiled display device that is readily accessible to visualization researchers and domain scientists alike. The LBNL Personal Display (PD) Wall differentiates itself from other LCD-matrix displays because its design minimizes cost and complexity while retaining the functionality of its more expensive tiled display brethren. The PD-Wall occupies the same amount of desktop area as a large flatscreen LCD display panel. LBNL will be publishing and distributing simple plans so that any laboratory or user site can construct their own copies of this device.

\section{Benefits}

The user community has been slow to adopt multiple-display technologies in their normal working environment. They are inconveniently located: users are disinclined to leave their office to make use of these expensive shared resources. In the case of NERSC, it makes little sense to create such a shared facility when most users are predominantly offsite. Remote NERSC users are also not likely to create their own local display wall facilities due to the complexity of these devices and the burden of supporting additional employees with the expertise to operate these devices.

Consequently, the expense and inconvenience of multiple-display technology has outweighed the benefit of high visual acuity for researchers.

The PD-Wall brings display-wall technology to a scientist's desktop with minimal expense, making it a more attractive and accessible component of their data analysis toolkit. The PD-wall does not require considerable skill or expertise to assemble, so it has the potential for wide adoption by offsite NERSC users using only modest resources. Pervasive adoption of this simplified display wall implementation makes it easier for visualization professionals to develop robust solutions that use this kind of display technology. Likewise, wider adoption of these new display technologies by the user community will actually assist the NERSC visualization group in their desire to offer more advanced applications and visualization technology to offsite users - an endeavour that is often constrained by the resources available to our users worksite. 
1. InteractiveMural: http://www.cs.umd.edu/ francois/Mural/

2. UMN InTENsity PowerWall:

http://www.lcse.umn.edu/sc99/SC99Intensitylnfo files/v3 document.htm

3. http://www.nas.nasa.gov/Groups/VisTech/hyperwall/

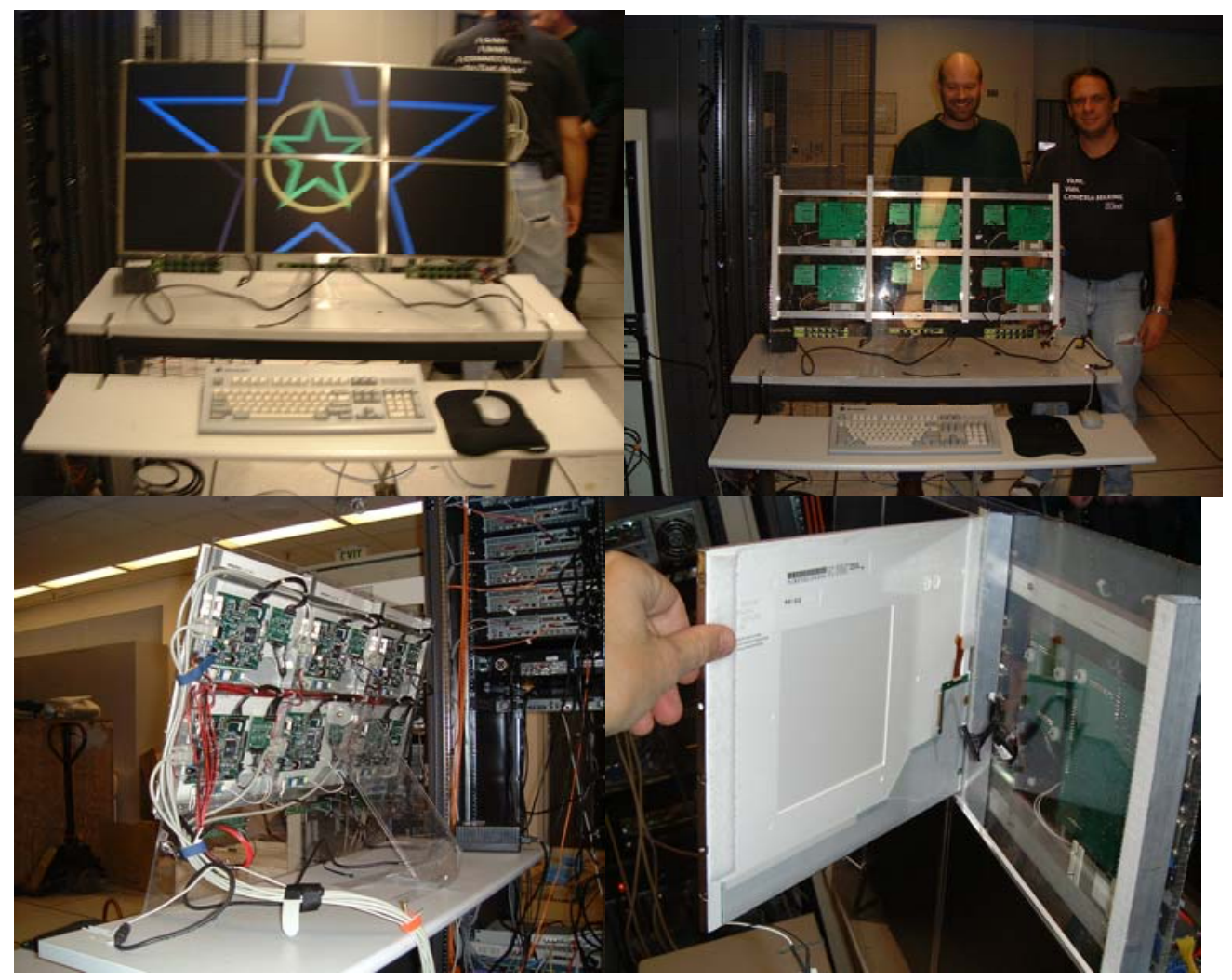

Figure 1: Images of the Personal Display Wall (PD-Wall). The upper left image shows the wall running the Distributed Memory Xserver (DMX) to create a single logical desktop from the multiple panels. The upper right image shows the simple aluminum and Plexiglas construction of the flatpanel. The lower left image shows the back of the display panel. The lower right image shows how the LCD's are affixed to the panel using Velcro - simplifying construction, assembly, and maintenance of the PD-Wall device. Each display of the PD-Wall is a single LCD laptop panel that can be purchased from an online vendor for about $\$ 200$. The total cost of our prototype - a $3 \times 2$ array - was less than $\$ 1500$, yet it provides an aggregate pixel resolution of $3072 \times 1536$.

\section{Contact}

John Shalf, LBNL/NERSC. jshalf@lbl.gov, 510-486-4508. 